\title{
Simulación FOTOVOLTAICA CONSIDERANDO PARÁMETROS DE INTEGRACIÓN EN EDIFICACIONES
}

\section{Photovoltaic SimUlation CONSIDERING BUILDING INTEGRATION PARAMETERS}

\author{
Ismael F. Izquierdo-Torres ${ }^{1, *}$, Mario G. Pacheco-Portilla ${ }^{1}$, \\ Luis G. González-Morales², Esteban F. Zalamea-León ${ }^{3}$
}

\section{Resumen}

Esta investigación calibra y valida un modelo de sistemas fotovoltaicos monocristalinos en la herramienta computacional System Advisor Model (SAM) para simulación de generación eléctrica, considerando las características meteorológicas en Cuenca (Ecuador), ciudad en altura próxima a la línea ecuatorial. Se obtiene el rendimiento eléctrico al desplegarse paneles fotovoltaicos de características específicas, con inclinaciones que responden a techumbres típicas locales y distintas orientaciones. Se calcula la eficiencia con mediciones in situ durante un período de 18 días, para que, con datos meteorológicos se calibre un archivo climático para el año 2016. Se estiman rendimientos anuales acordes con la inclinación y orientación, y las características técnicas de los fotovoltaicos. Se detectan pérdidas por acumulación de suciedad e incremento de temperatura de las placas. Se valida el modelo mediante una regresión lineal, al comparar los valores simulados con los datos obtenidos de mediciones in situ de un panel en posición horizontal. Los resultados indican una pérdida promedio de eficiencia de $2,77 \%$ por condiciones de suciedad y de hasta el $30 \%$ por incremento de temperatura.

\begin{abstract}
This research calibrates and validates a model for monocrystalline photovoltaic systems in SAM (System Advisor Model) for power generation simulation, considering the meteorological characteristics of Cuenca, Ecuador, close to the equatorial line. The electrical performance is calculated by arranging photovoltaic systems with specific characteristics, with inclinations that respond to conventional local roofing and different orientations. Efficiency is calculated with in-situ measurements over a period of 18 days. Meteorological data were used to calibrate a weather file for the year 2016. Annual yields are estimated according to inclination and orientation, and technical characteristics of the photovoltaic system. Losses are detected due to dirt accumulation and increase in temperature of the panels. The model is validated by linear regression, by comparing the simulated values with the data obtained from in-situ measurements of a reference panel deployed horizontally. The results show an average efficiency loss of $2,77 \%$ for dirt conditions and up to $30 \%$ for temperature increases.
\end{abstract}

\footnotetext{
${ }_{1, *}$ Facultad de Ciencias Químicas, Universidad de Cuenca, Ecuador. Autor para correspondencia ismael.izquierdo.torres@ucuenca.edu.ec, (D) http://orcid.org/0000-0003-0728-8978, (D) http://orcid.org/0000-0003-4107-4594

${ }^{2}$ Departamento de Ingeniería Eléctrica, Electrónica y Telecomunicaciones, Universidad de Cuenca, Ecuador. (1) http://orcid.org/0000-0001-9992-3494

${ }^{3}$ Facultad de Arquitectura y Urbanismo, Universidad de Cuenca, Ecuador. (D) http://orcid.org/0000-0001-5551-5026

Recibido: 06-07-2018, aprobado tras revisión: 05-11-2018

Forma sugerida de citación: Izquierdo-Torres, I. F.; Pacheco-Portilla, M. G; González-Morales, L. G. y ZalameaLeón, E. F. (2019). «Simulación fotovoltaica considerando parámetros de integración en edificaciones». INGENIUS. $\mathrm{N}{ }^{\circ}$ 21, (enero-junio). pp. 21-31. DOI: https://doi.org/10.17163/ings.n21.2019.02.
} 
La validación del modelo mostró un coeficiente de determinación $\mathrm{R}^{2}=0,996$ y un RMSE normalizado de $8,16 \%$. Se concluye, además, que por la latitud particular del sitio en estudio, a diferencia de la mayor parte del planeta, la disposición de paneles fotovoltaicos en cualquier orientación considerando pendientes bajas, no reduce significativamente el rendimiento en la generación de energía eléctrica anual.

Palabras clave: energías renovables, monocristalino, SAM, simulación fotovoltaica.
The validation of the model showed a determination coefficient $\mathrm{R}^{2}=0,996$ and a normalized Root Mean Square Error (RMSE) of $8,16 \%$. It is concluded that, because of the particular latitude of the study site, unlike most of the planet, the provision of photovoltaic panels in any orientation considering low slopes, does not significantly reduce the annual power generation performance.

Keywords: Monocrystalline, Photovoltaic Simulation, SAM, Renewable Energies. 


\section{Introducción}

Las crecientes demandas energéticas y procesos antropogénicos causantes del cambio climático hacen necesario el recurrir a fuentes alternativas de energía limpia, renovable y con potencial de expansión con menor impacto [1]. Una condición ideal es que puedan, además, desplegarse en edificaciones y contextos urbanos $[2,3]$. La tecnología solar fotovoltaica es una de las principales alternativas para afrontar decididamente la problemática energética global, tanto por el potencial existente para su expansión, sumado a costos cada vez más convenientes $[4,5]$.

Por condiciones de localización, Ecuador está en una latitud en la que el potencial solar es particular e importante acorde a estudios en estas locaciones $[6,7]$. Ecuador es un país en desarrollo con creciente consumo energético y estacionalidad poco variable a lo largo del año [8,9], en consecuencia, existen niveles altos de irradiación relativamente constantes [10]; además, posee un gran potencial de aprovechamiento de energía solar como alternativa para la efectiva disminución de contaminantes atmosféricos y los efectos globales del cambio climático [11-13].

Sin embargo, es necesario el determinar el potencial de generación energética variable en cada entorno urbano, pues cada ciudad posee características propias en recursos y demandas energéticas particulares [11], [14], como antesala para establecer normativas y regulaciones urbanísticas [15] y de infraestructura energética [16]. La agencia internacional IRENA (International Renewable Energy Agency), recomienda el aprovechamiento de las grandes áreas de envolventes y techumbres para el emplazamiento de sistemas de captación de energía como complemento a medidas de eficiencia energética en edificaciones, implicando la producción de energía de forma distribuida [12].

En el caso de tecnologías solares fotovoltaicas o solar térmica deben considerarse aspectos de impacto urbanístico, por ello la opción es que las superficies de captación estén coplanares en la edificación; formando parte de la envolvente [17]. Además, deben desplegarse en coherencia con los consumos, en función de cada edificación [18].

Actualmente, están en desarrollo paneles fotovoltaicos (desde ahora PV) que son elementos constructivos arquitectónicos con capacidad de generación energética, como placas de revestimiento, vidrios, filtros solares e incluso tejas que, respetando la geometría y siendo parte de la envolvente, cumplen el concepto de integración arquitectónica [PV Building Integrated Photovoltaics (BIPV)] [19-21]. Este trabajo pretende determinar la eficiencia eléctrica $\mathrm{PV}$ de placas monocristalinas acorde a inclinación y orientación, para así prever una base metodológica para desarrollar un modelo de predicción del desempeño y rendimiento de otras tecnologías PV, adaptables o integrables en envolventes, lo cual implica medir rendimientos en distintas orientaciones e inclinaciones.

Para ciudades como Cuenca, no existen criterios claros respecto a parámetros de inclinación y orientación óptimas PV, asumiéndose como adecuada cualquier inclinación cercana a la horizontal y orientada contraria a su latitud [22]. Sin embargo, en dicha configuración no se contemplan criterios de adaptación coplanar a edificaciones, así como el efecto de la acumulación de suciedad en el rendimiento del sistema, cuyas pérdidas documentadas varían entre el $5 \%$ [23] y $35 \%$ [24].

En instalaciones PV, a más de estos factores, la temperatura superficial de los módulos fotovoltaicos también afecta a la eficiencia del sistema [25-28]. Adicionalmente, es necesario considerar los aspectos económicos y las demandas energéticas, para así maximizar la producción en los momentos de mayor demanda o de mayor costo energético [29].

El presente estudio plantea una metodología para obtener un modelo de simulación en el programa SAM (System Advisor Model) [30] para estimar la producción eléctrica de paneles PV. Se aplica para ello una validación en placas PV de tipo monocristalino; en este modelo se estudian condiciones y consecuencias de orientación, inclinación y pérdidas de eficiencia por acumulación de suciedad y por incrementos de temperatura, en el contexto particular planteado, calibrándose el modelo acorde a estos parámetros. Para ello es necesario el ajuste del modelo en SAM, y del archivo climático correspondiente. Se obtiene un modelo válido para simulaciones con respecto a valores reales de producción [31], evitando recurrir a mediciones in situ por períodos de un año o más para determinar el rendimiento PV, o usar otros modelos con mayor incertidumbre y que no sean de acceso libre para el usuario. De esta manera, la metodología de calibrado podría ser replicada en otras locaciones.

\section{Materiales y métodos}

El presente estudio se enfoca en la calibración y validación de un modelo de simulación de un sistema fotovoltaico monocristalino a partir de cortos períodos de medición in situ y, además, un archivo climático de la localidad, generado en formato legible por el software (SAM CSV). Se consideran también parámetros de pérdidas de desempeño debido a la acumulación de suciedad y por incremento de temperatura en función del clima e irradiancia global.

Las mediciones in situ se llevaron a cabo durante el mes de diciembre de 2016 y enero de 2017. Para ello se instalaron tres paneles PV monocristalinos junto a una estación meteorológica en la ubicación $\left(-2,901691^{\circ} \mathrm{S}\right.$, $\left.-79,010151^{\circ} \mathrm{E}\right)$. Cada panel tiene una potencia nominal de $100 \mathrm{~W}$, cuyas dimensiones son $0,54 \mathrm{~m}$ de ancho y 
1,2 $\mathrm{m}$ de largo, de 36 celdas (Figura 1), conectados eléctricamente a un conjunto de resistencias empleadas como cargas que variaban entre 1 y $100 \Omega$ en función de la irradiancia recibida en los paneles para obtener su máxima potencia, y al equipo de medición HIOKI PW 3337-03 (Figura 2). Se procede a la toma de datos en corriente continua de voltaje, corriente y potencia en intervalos de cinco minutos, que posteriormente fueron transformados a intervalos horarios.

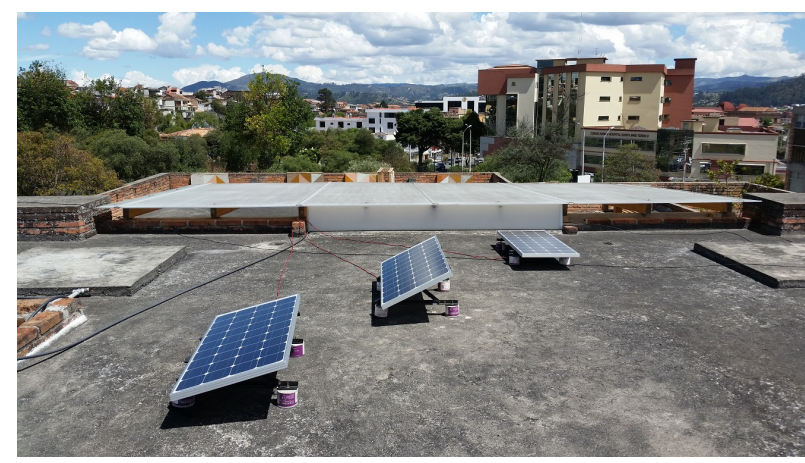

Figura 1. Paneles solares monocristalinos a distintas inclinaciones.

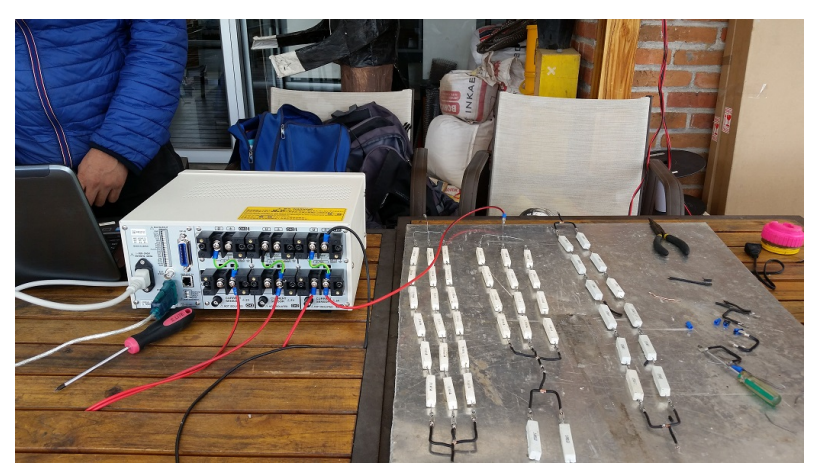

Figura 2. Equipo HIOKI PW 3337-03 y resistencias de carga.

Las mediciones in situ se realizaron basándose en la inclinación y orientación de los paneles, durante 12 días del mes de diciembre de 2016 entre las 07:30 y las 17:00. Se obtuvieron datos del panel horizontal y datos de paneles ubicados a $14,00^{\circ}, 18,26^{\circ}$ y $26,56^{\circ}$ de inclinación (en todas las orientaciones: $\mathrm{N}, \mathrm{S}, \mathrm{E}, \mathrm{O}$ ) con lo que se valida el modelo en SAM. Las inclinaciones consideradas responden a pendientes características de techumbres en edificaciones de baja altura y vivienda en la ciudad de Cuenca [32]. Para la medición de rendimiento según inclinación y orientación, se utilizó la metodología mostrada en la Tabla 1.

Posteriormente se calculó la eficiencia en función de la Ecuación (1):

$$
\eta=\frac{P}{E \times A c} \times 100
$$

Tabla 1. Variación paramétrica para medir el desempeño de paneles monocristalinos en base a su inclinación y orientación

\begin{tabular}{cccc}
\hline \multicolumn{4}{c}{ Este } \\
\hline Día & Panel 1 & Panel 2 & Panel 3 \\
1 & $0^{\circ}$ & $14,00^{\circ}$ & $18,26^{\circ}$ \\
2 & $0^{\circ}$ & $18,26^{\circ}$ & $26,56^{\circ}$ \\
3 & $0^{\circ}$ & $26,56^{\circ}$ & $14,00^{\circ}$ \\
\hline \multicolumn{4}{c}{ Sur } \\
\hline Día & Panel 1 & Panel 2 & Panel 3 \\
4 & $0^{\circ}$ & $14,00^{\circ}$ & $18,26^{\circ}$ \\
5 & $0^{\circ}$ & $18,26^{\circ}$ & $26,56^{\circ}$ \\
6 & $0^{\circ}$ & $26,56^{\circ}$ & $14,00^{\circ}$ \\
\hline \multicolumn{4}{c}{ Oeste } \\
\hline Día & Panel 1 & Panel 2 & Panel 3 \\
7 & $0^{\circ}$ & $14,00^{\circ}$ & $18,26^{\circ}$ \\
8 & $0^{\circ}$ & $18,26^{\circ}$ & $26,56^{\circ}$ \\
9 & $0^{\circ}$ & $26,56^{\circ}$ & $14,00^{\circ}$ \\
\hline \multicolumn{4}{c}{ Norte } \\
\hline Día & Panel 1 & Panel 2 & Panel 3 \\
10 & $0^{\circ}$ & $14,00^{\circ}$ & $18,26^{\circ}$ \\
11 & $0^{\circ}$ & $18,26^{\circ}$ & $26,56^{\circ}$ \\
12 & $0^{\circ}$ & $26,56^{\circ}$ & $14,00^{\circ}$ \\
\hline
\end{tabular}

Donde $\eta$ es la eficiencia de los paneles, $P$ es la potencia de salida, $E$ es la irradiancia del sol, y $A c$ es el área de captación o área del panel. De las tres inclinaciones propuestas se seleccionó la mejor de cada orientación y se realizó una medición adicional durante tres días en el mes de enero de 2017 para determinar la configuración óptima para esta época del año (tanto en inclinación y orientación), siguiendo la metodología indicada en la Tabla 2.

Tabla 2. Configuración óptima de paneles monocristalinos para el mes de enero 2017

\begin{tabular}{cccc}
\hline Día & Panel 1 & Panel 2 & Panel 3 \\
\hline 1 & S 26,56 & E 14,00 & N 18,26 \\
2 & S 26,56 & N 18,26 & O 18,26 \\
3 & S 26,56 & O 18,26 & E 14,00 \\
\hline
\end{tabular}

De las cuatro configuraciones analizadas, se seleccionó la óptima según los valores de producción energética promedio durante los días de medición.

Por otra parte, para estimar las pérdidas energéticas por la acumulación de suciedad, se realizaron mediciones semanales del desempeño PV, empezando el 11 de enero y culminando el 1 de febrero de 2017, ubicando la orientación e inclinación determinadas como óptimas. Para este caso uno de los paneles cumplió la función de control y recibió limpieza durante los días de medición, mientras que los dos restantes no 
tuvieron ninguna intervención; sin embargo, debido a que los eventos de precipitación en Cuenca son frecuentes, los paneles que no recibieron mantenimiento de forma manual, presentaron cambios en la superficie por efecto de la precipitación, que limpiaba los paneles durante los días de estudio. Es necesario considerar que el clima local muestra alta pluviosidad, existiendo precipitaciones durante todo el año en mayor o menor medida; siendo extraños los períodos sin lluvias por más de un mes [33].

Para la estimación de pérdidas por incremento de temperatura, se midió la salida de potencia de los paneles monocristalinos y la temperatura superficial de los mismos; posteriormente se calculó la eficiencia mediante la Ecuación 1 con los datos de irradiancia obtenidos de la estación meteorológica, y se establecieron valores promedio de pérdida de eficiencia por rangos de irradiancia. Entonces, con los datos obtenidos de pérdidas promedio de desempeño por acumulación de suciedad y por rangos de irradiancia se procedió a calibrar el modelo en SAM. La información incorporada y tomada de lecturas en sitio, fue un archivo climático con información horaria de la localidad, durante el año 2016 generado de acuerdo con valores horarios de radiación directa $\left(\mathrm{W} / \mathrm{m}^{2}\right)$, radiación difusa $\left(\mathrm{W} / \mathrm{m}^{2}\right)$, radiación global $\left(\mathrm{W} / \mathrm{m}^{2}\right)$, humedad relativa $(\%)$, ángulo cenital $\left(^{\circ}\right)$, presión atmosférica (mbar), temperatura ambiente $\left({ }^{\circ} \mathrm{C}\right)$, precipitación $(\mathrm{mm})$, dirección y velocidad del viento $\left({ }^{\circ} \mathrm{y} \mathrm{m} / \mathrm{s}\right)$. Así, el archivo climático del sitio de estudio, no es el resultado de la interpolación de valores de variables climáticas entre dos localidades, que ocasiona un mayor grado de incertidumbre en las predicciones de los modelos, sino que la validación de resultados se realiza con una comparación real suscitada entre lecturas de producción PV y condiciones climáticas detectadas al instante.

Adicionalmente, se incorporó a las especificaciones del software, datos técnicos de los paneles PV utilizados, los cuales son mostrados en la Tabla 3.

Tabla 3. Especificaciones de panel fotovoltaico simulado

\begin{tabular}{|c|c|c|c|}
\hline \multicolumn{4}{|c|}{ Especificaciones } \\
\hline $\begin{array}{l}\text { Tipo de } \\
\text { celda }\end{array}$ & $\begin{array}{c}\text { Silicio } \\
\text { monocristalino }\end{array}$ & Voc & $21,6 \mathrm{~V}$ \\
\hline $\begin{array}{l}\text { Área del } \\
\text { módulo }\end{array}$ & $0,645 \mathrm{~m}^{2}$ & $\begin{array}{c}\text { Coeficiente de } \\
\text { Temperatura Voc }\end{array}$ & $-0,38 \% /{ }^{\circ} \mathrm{C}$ \\
\hline NOCT & $46^{\circ} \mathrm{C}$ & $\begin{array}{l}\text { Coeficiente de } \\
\text { Temperatura Isc }\end{array}$ & $0,1 \% /{ }^{\circ} \mathrm{C}$ \\
\hline Vmp & $17,3 \mathrm{~V}$ & $\begin{array}{c}\text { Coeficiente de } \\
\text { Temperatura MPP }\end{array}$ & $-0,41 \% /{ }^{\circ} \mathrm{C}$ \\
\hline $\operatorname{Imp}$ & $5,78 \mathrm{~A}$ & $\begin{array}{c}\text { Número de celdas } \\
\text { en serie }\end{array}$ & 36 \\
\hline
\end{tabular}

En este estudio se definen aspectos relacionados con el procesamiento de energía, por lo que, para el modelo se consideró el punto de máxima potencia del panel para cada estimación. Las pérdidas en el sistema fueron establecidas sobre la base de lecturas de eficiencia obtenidas in situ por rangos de irradiancia en intervalos de $200 \mathrm{~W} / \mathrm{m}^{2}$ y por pérdidas debido a la acumulación de suciedad, que para efectos de cálculo se utiliza como base una pérdida de $5 \%$ establecida por defecto dentro del programa. Con los datos de rendimientos simulados, se generó una base de datos comparable con los valores medidos in situ del panel horizontal y se calcularon las métricas: Coeficiente de determinación $\mathrm{R}^{2}$ (Ecuación 2), raíz del error cuadrático medio (RMSE) (Ecuación 3) y su valor normalizado, error de sesgo medio (MBE) (Ecuación 4) así como su valor normalizado; finalmente, se establece el intervalo de confianza de 90 \% (IC 90 \%) (Ecuación 5 ), para intervalos horarios, recomendados por algunos estudios de validación de SAM [34,35], para esta época del año. Los valores del RMSE y MBE fueron normalizados con respecto al valor máximo reportado en las mediciones in situ.

$$
\begin{gathered}
R^{2}=\frac{\sum_{i}^{N}\left(S A M_{i}-M_{i} \text { dido } o_{a v g}\right)^{2}}{\sum_{i}^{N}\left(S A M_{i}-S A M_{a v g}\right)^{2}} \\
R M S E=\sqrt{\frac{\sum_{i}^{N}\left(S A M_{i}-M_{e d i d o}\right)^{2}}{N}} \\
M B E=\frac{\sum_{i}^{N}\left(S A M_{i}-\text { Medido }_{i}\right)^{2}}{N} \\
I C_{90 \%}=1.645 \times\left[S t d\left(S A M_{i}-\text { Medido }_{i}\right)\right]
\end{gathered}
$$

\section{Resultados y discusión}

De acuerdo con la variación paramétrica para medir el desempeño y cálculos de eficiencia en el mes de diciembre, se obtuvieron los resultados mostrados de mayor a menor en la Tabla 4.

Tabla 4. Mejores inclinaciones por orientación

\begin{tabular}{cc}
\hline Orientación & Mejor inclinación \\
\hline Este & $14,00^{\circ}$ \\
Sur & $26,56^{\circ}$ \\
Oeste & $18,26^{\circ}$ \\
Norte & $18,26^{\circ}$ \\
\hline
\end{tabular}

Los resultados de la Tabla 4 se deben a la posición que tiene el Sol en esta época del año (cercana al solsticio), por lo que la orientación sur supera a la orientación norte en cualquier inclinación; sin embargo, la orientación este a $14^{\circ}$ otorgó una mayor eficiencia debido a las mañanas con alta irradiancia y tardes de alta nubosidad, por lo que fue seleccionada para el posterior análisis de influencia de suciedad.

En el análisis de influencia de suciedad, durante la primera semana de mediciones, no se presentaron 
pérdidas en el desempeño debido a la cantidad de precipitación acumulada semanal (41,8 $\mathrm{mm})$; a la segunda semana de monitoreo se registró un total de precipitación de $6 \mathrm{~mm}$, que como consecuencia resulta en manchas de suciedad en la superficie y a una disminución poco significativa en la eficiencia de los paneles $(0,7 \%)$.

En la tercera semana no existieron eventos de lluvia, siendo evidente la presencia de material depositado en la superficie de los paneles; las mediciones correspondientes mostraron una reducción promedio de eficiencia de $2,77 \%$, un máximo de $3,68 \%$ y un mínimo de $1,87 \%$. Una semana después de esta medición, la precipitación acumulada $(13,4 \mathrm{~mm})$ evidencia la remoción de una cantidad significativa de partículas en su superficie, haciendo que las pérdidas por esta sean despreciables. Al ser muy bajo el valor promedio de pérdida de eficiencia, para la simulación en SAM, se optó por el valor predefinido de $5 \%$. Como se mencionó, en la localidad no existen períodos largos sin lluvia, característica de las ciudades andinas en altura.

A partir de los datos generados se pudo establecer una base comparativa para calibrar y validar el modelo en SAM, el cual se generó a partir de los parámetros especificados en la metodología. Se compararon los resultados del modelo con los obtenidos con mediciones in situ del panel horizontal, de esta comparación se calcularon datos estadísticos de interés en la Tabla 5 .

Tabla 5. Métricas calculadas

\begin{tabular}{ccc}
\hline Métrica & Valor & Unidad \\
\hline $\mathrm{R}^{2}$ & 0,996 & - \\
$\mathrm{RMSE}$ & 5,263 & $\mathrm{~W}$ \\
$\mathrm{NRMSE}$ & 8,156 & $\%$ \\
$\mathrm{MBE}$ & $-1,04$ & $\mathrm{~W}$ \\
$\mathrm{NMBE}$ & $-1,616$ & $\%$ \\
$\mathrm{IC} 90 \%$ & 8,522 & $\%$ \\
\hline
\end{tabular}

De forma gráfica, los datos in situ y los obtenidos por el modelo se muestran en la Figura 3. Se evidencia una fuerte correlación lineal respaldada en el valor del coeficiente de determinación $\mathrm{R}^{2}$ encontrado. Los valores de RMSE y NRMSE indican el grado de error que posee la estimación frente a lecturas reales, y en este caso particular son bastante aceptables [36].

Para determinar la subestimación o sobrestimación del modelo, es empleado el error de sesgo medio o MBE (Mean Bias Error) y su valor normalizado denotando que el modelo subestima el desempeño del sistema en un 1,61\%. La Figura 4 muestra el comportamiento real del sistema y el estimado por el modelo en SAM para 12 días de medición, tomando como referencia validaciones en cortos períodos efectuadas en TRNSYS por [36,37], donde sus valores máximos de generación son tendenciales, observándose en general una ligera subestimación del modelo. Adicionalmente, existen estudios donde se analiza la resolución temporal en intervalos menores a una hora [38] y otro donde se comparan valores simulados e in situ en un período de dos días [39].

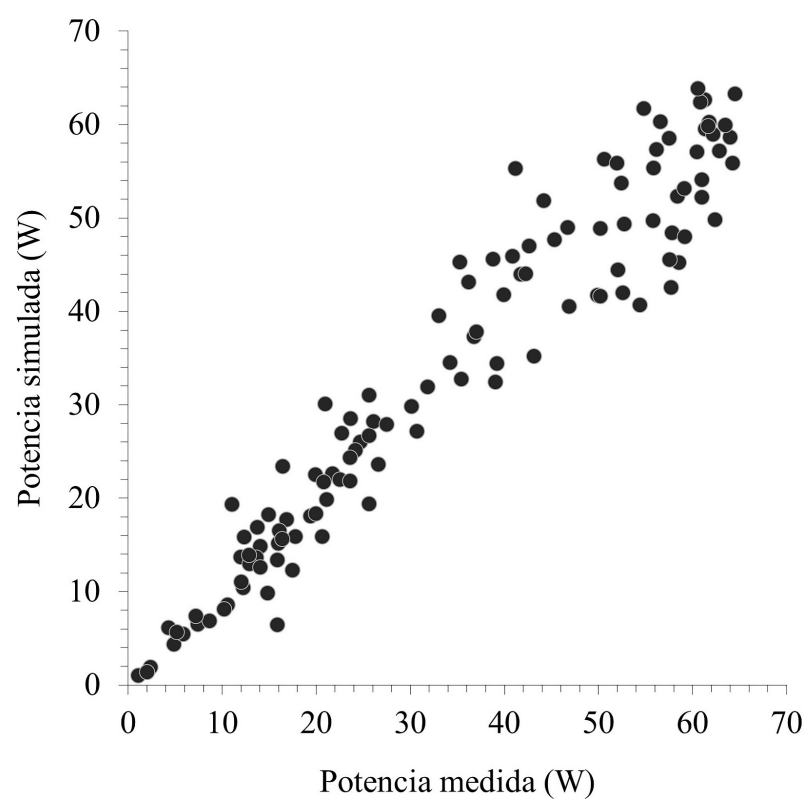

Figura 3. Gráfica de dispersión de potencia medida y simulada.

El valor del IC $90 \%$ (intervalo de confianza de $90 \%$ ) de la Tabla 5, indica que el $90 \%$ de los valores simulados se encuentran entre un $\pm 8,522 \%$ de los valores medidos in situ, valor muy próximo al $\pm 8 \%$, con el que se han validado siete casos en estudios referenciales con SAM [34]. Cabe recalcar que en una simulación anual este valor se reduce, ajustándose mejor al modelo lineal y presentando mayor validez.

Adicionalmente, la estimación de temperatura de las celdas PV por parte del modelo difiere de las mediciones realizadas in situ. Para compararlas se empleó la temperatura promedio anual de la base de datos del modelo ya que para los mismos niveles de radiación la temperatura parece comportarse de manera similar a lo largo del año; mientras que para los datos in situ se ocuparon valores puntuales de un día de medición. Se observa una diferencia promedio importante de $13,33^{\circ} \mathrm{C}$ de los datos mostrados en la Tabla 6 , y las celdas sin valores denotan que, durante el día, los valores de radiación no estuvieron dentro de estos intervalos. Se asume, además, que la diferencia puede ser mayor ya que el modelo estima la temperatura de las celdas, mientras que las mediciones se las realizaron sobre el vidrio del panel que es inferior a la temperatura superficial de las celdas [40], esta variación de temperatura también puede atribuirse a la intensidad de rayos UV, por tratarse de una locación en altura [41]. Por ello, se recomendaría realizar lecturas en laboratorio para corroborar este incremento de temperatura en la superficie de las celdas. 


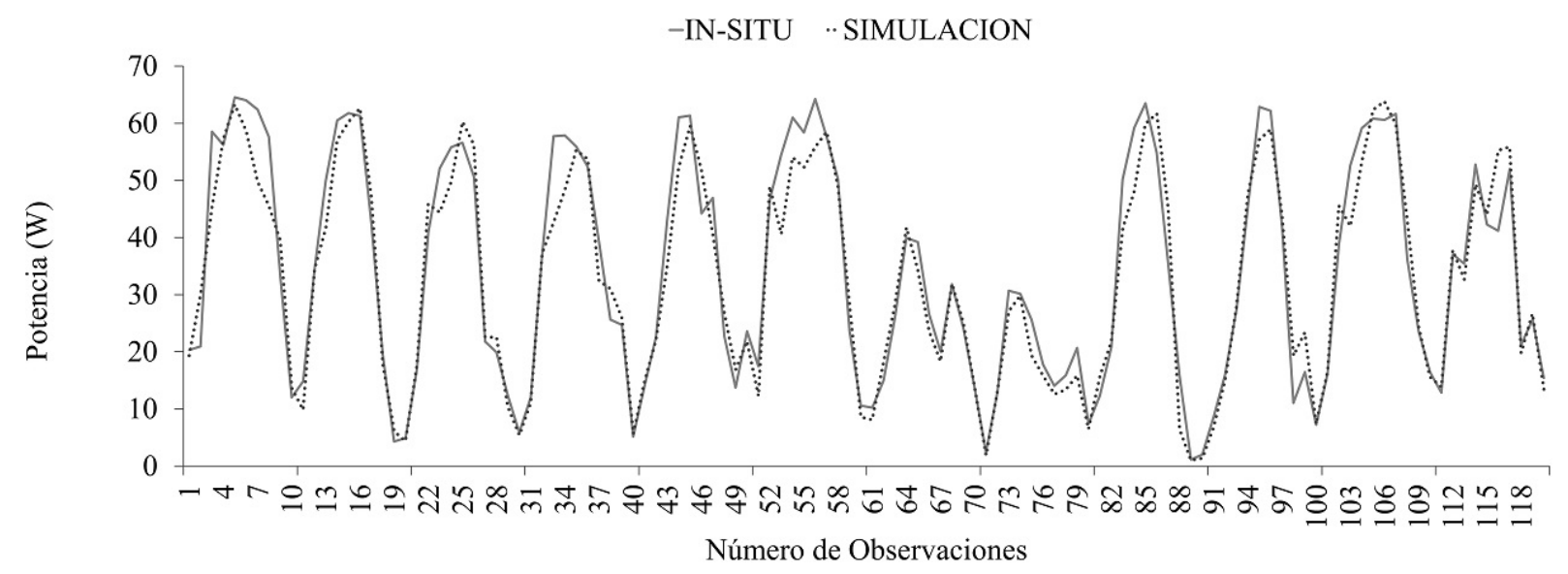

Figura 4. Comparación de desempeño.

Tabla 6. Comparación de la temperatura simulada y la temperatura medida

\begin{tabular}{ccc}
\hline $\begin{array}{c}\text { Irradiancia } \\
\left(\mathbf{W} / \mathbf{m}^{2}\right)\end{array}$ & $\begin{array}{c}\text { Temperatura } \\
\text { promedio } \\
\text { anual }\left({ }^{\circ} \mathbf{C}\right)\end{array}$ & $\begin{array}{c}\text { Temperatura } \\
\text { Medida }\left({ }^{\circ} \mathbf{C}\right)\end{array}$ \\
\hline $0-200$ & 15,81 & - \\
$200-400$ & 21,05 & 33,5 \\
$400-600$ & 24,56 & - \\
$600-800$ & 27,02 & 40 \\
$800-1000$ & 30,33 & 43 \\
$1000-1200$ & 34,08 & 49,33 \\
\hline
\end{tabular}

Con los valores de estas métricas se determinó que el modelo es válido. Por ello es factible estimar la producción energética en períodos más prolongados, con la disponibilidad del archivo climático. Al realizar una simulación anual expresada en $\mathrm{kWh} / \mathrm{año}$ para un módulo de $100 \mathrm{~W}$, considerando los parámetros de orientación e inclinación, se obtienen los rendimientos señalados en la Tabla 7.

Así la situación que presenta mayor generación es la disposición horizontal; sin embargo, no se ajusta a un posible despliegue en techumbres inclinadas locales, además, no es una alternativa recomendable para aprovechamiento de limpieza pluvial.

Tabla 7. Producción energética anual (kWh/año)

\begin{tabular}{ccccc}
\hline Ángulo & Norte & Sur & Este & Oeste \\
\hline $\mathbf{0}^{\circ}$ & 119,16 & & & \\
$\mathbf{1 4}^{\circ}$ & 116,5 & 116,94 & 117,21 & 116,24 \\
$\mathbf{1 8 , 2 6}^{\circ}$ & 114,66 & 115,24 & 115,63 & 114,35 \\
$\mathbf{2 6 , 6}^{\circ}$ & 109,76 & 110,64 & 111,35 & 109,5 \\
\hline
\end{tabular}

En la Tabla 7 se establece una particularidad interesante respecto a la orientación y pendiente de PV en el contexto de estudio. La máxima producción efectivamente se alcanza con la disposición horizontal, pero supera en apenas un 8,8\% en generación anual respecto a la mínima producción establecida en este estudio (orientación oeste con $26,56^{\circ}$ de inclinación), pero, por las limitaciones mencionadas ello implica "no recomendable" el despliegue horizontal. En la comparación de rendimiento de los casos inclinados y orientados, se observa que a menor pendiente efectivamente se logra una mejor producción promedio, teniendo 5,8\% más de energía en el despliegue inclinado a $14^{\circ}$ respecto al inclinado $26,56^{\circ}$; y $4,2 \%$ con inclinación de $18^{\circ}$ respecto a inclinado en $26,56^{\circ}$. En análisis de rendimientos puntuales, la máxima producción se observa en orientación este con $14^{\circ}$ de inclinación y la mínima con orientación oeste y $26,56^{\circ}$ de inclinación, siendo más eficiente la primera disposición en un 7,0\% y los demás rendimientos son condiciones intermedias. Las pérdidas medidas en este estudio son menores, considerando por ejemplo estimaciones de rendimiento PV efectuado en condición de clima estacional moderado ( $36^{\circ}$ de latitud) en donde un desvío de $90^{\circ}$ respecto la orientación óptima significa una reducción de rendimiento PV de $17 \%$ [42]; lógicamente una orientación con mayor desviación contraria al recorrido solar y en latitudes más alejadas al ecuador supondrían una reducción de rendimiento PV muy superiores. La explicación para el rendimiento superior con orientación este es por tendencia a mañanas con menor nubosidad.

Para el caso de valores mensuales es observable una diferencia más marcada, entre $7,33 \mathrm{kWh} /$ mes en el mes de julio (mes con mínima irradiación) y 12,19kWh/mes en noviembre (máxima irradiación) respectivamente (Figura 5).

Esto refleja la estabilidad de producción existente en el contexto, frente a estudios de abastecimiento $\mathrm{PV}$ desarrollados en clima estacional moderado $\left(38^{\circ}\right.$ latitud sur), en donde se observa que la generación se puede triplicar en meses de verano respecto a los invernales [43]; y en climas con estacionalidad marcada, como Helsinki (Finlandia), en latitud extrema ( $60^{\circ}$ latitud norte) en donde se observa que la producción en 
verano excede en más de diez veces respecto a la esperada en invierno. Esto se traduce en una ventaja obvia de las zonas ecuatoriales ya que sería más adaptable frente a demandas de edificaciones y urbanas.

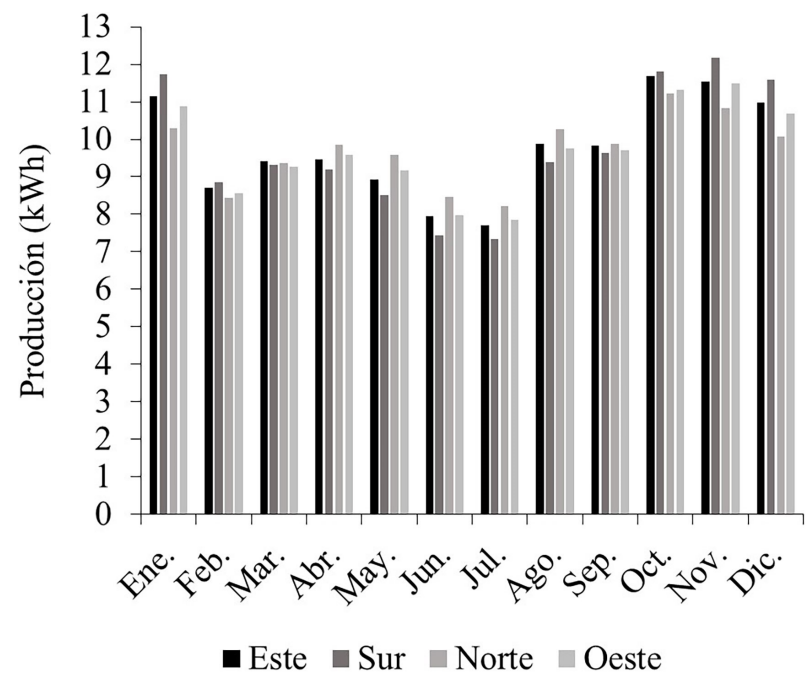

Figura 5. Producción mensual estimada mediante simulación en SAM.

\section{Conclusiones}

Un paso importante para poder desarrollar el potencial de generación de energía renovable de una ciudad, es validar las herramientas que permitan reducir incertidumbres de estimaciones y simulaciones, en este caso de producción PV. Este estudio muestra que, mediante ajustes puntuales en una serie de parámetros, el modelo SAM puede emplearse con baja incertidumbre en la ciudad de Cuenca (Ecuador), entregando un modelo fiable para proyectar una posible generación eléctrica PV. Para otras localidades, se puede optar por una calibración similar a la empleada en este estudio, y con validación de lecturas de rendimiento in situ se puede asegurar que el modelo sea válido.

Entre otros resultados obtenidos, se ha medido la pérdida de eficiencia por acumulación de suciedad, que en promedio es de $2,77 \%$, siendo no significativo y que permitiría prescindir de una limpieza recurrente de los paneles PV, pues la continuidad de precipitaciones locales es suficiente para recuperar la eficiencia. Por otra parte, en el caso de las pérdidas por efecto de la temperatura se miden como valores elevados bajo presencia de alta irradiación, explicable por condiciones constructivas de las celdas o por la cantidad de irradiación con elevados índices de rayos UV, por lo que este fue un parámetro relevante durante la calibración del modelo.

El valor de las métricas estadísticas del modelo frente a lecturas en sitio fue de $\mathrm{R}^{2}=0,996$, $\mathrm{NRMSE}=8,156$ y $\mathrm{NMBE}=-1,616 \%$ demostrando que, a pesar de preverse una subestimación, es adecuado para emplearlo en predicciones de escenarios futuros en simulaciones anuales, pues guarda una tendencia lineal marcada con respecto a los valores in situ. Se desarrolla una metodología y se obtiene una herramienta validada, útil para la estimación de generación eléctrica mediante paneles solares monocristalinos.

En cuanto a rendimientos detectados en el contexto específico, el emplazamiento de sistemas PV tiene una generación anual mayor al disponerse de cara al este con pendiente cercana a la horizontal, pero sin diferencias significativas respecto al resto de orientaciones con pendientes similares; esto es atribuible a la ubicación geográfica del área de estudio que presenta los niveles estables de irradiancia a lo largo del año. También, a pesar de observarse que, dependiendo de la inclinación y orientación, es esperable que cerca de los meses de junio-julio se produzca aproximadamente un $40 \%$ menos de electricidad (mes de mínima irradiación) frente a noviembre (mes de máxima irradiación), la producción sería mucho más estable respecto a otras latitudes en balance anual, y con ello adaptable a una potencial conexión a red inteligente o Smart Grid en generación distribuida o a almacenamiento para autoconsumo.

\section{Referencias}

[1] Z. A. Elum and A. S. Momodu, "Climate change mitigation and renewable energy for sustainable development in Nigeria: A discourse approach," Renewable and Sustainable Energy Reviews, vol. 76, pp. 72-80, 2017. [Online]. Available: https://doi.org/10.1016/j.rser.2017.03.040

[2] A. Grubler, X. Bai, T. Buettner, S. Dhakal, D. J. Fisk, T. Ichinose, J. E. Keirstead, G. Sammmer, D. Satterthwaite, N. B. Schulz et al., Global Energy Assessment - Toward a Sustainable Future. International Institute for Applied Systems Analysis and Cambridge University., 2012, ch. Urban energy systems, pp. 1307-1400. [Online]. Available: https://goo.gl/rVdsU6

[3] A. Barragán-Escandón, J. Terrados-Cepeda, and E. Zalamea-León, "The role of renewable energy in the promotion of circular urban metabolism," Sustainability, vol. 9, no. 12, 2017. [Online]. Available: https://doi.org/10.3390/su9122341

[4] A. K. Shukla, K. Sudhakar, P. Baredar, and R. Mamat, "Solar PV and BIPV system: Barrier, challenges and policy recommendation in India," Renewable and Sustainable Energy Reviews, vol. 82, pp. 3314-3322, 2018. [Online]. Available: https://doi.org/10.1016/j.rser.2017.10.013 
[5] J. Byrne, J. Taminiau, J. Seo, J. Lee, and S. Shin, "Are solar cities feasible? a review of current research," International Journal of Urban Sciences, vol. 21, no. 3, pp. 239-256, 2017. [Online]. Available: https://doi.org/10.1080/12265934.2017.1331750

[6] M. Nadim, M. R. H. Rashed, A. Muhury, and S. M. Mominuzzaman, "Estimation of optimum tilt angle for PV cell: A study in perspective of Bangladesh," in 2016 9th International Conference on Electrical and Computer Engineering (ICECE), Dec 2016, pp. 271-274. [Online]. Available: https://doi.org/10.1109/ICECE.2016.7853908

[7] P. Fitriaty and Z. Shen, "Predicting energy generation from residential building attached Photovoltaic Cells in a tropical area using 3D modeling analysis," Journal of Cleaner Production, vol. 195, pp. 1422-1436, 2018. [Online]. Available: https://doi.org/10.1016/j.jclepro.2018.02.133

[8] B. Trewin, State of the tropics. James Cook University, 2014, ch. The climates of the Tropics, and how they are changing, pp. 39-51. [Online]. Available: https://goo.gl/hH8pv1

[9] A. Bykerk-Kauffman. (2018) Seasons and why the equator is warmer than the poles. Pedagogy in Action the SERC portal for Educators. Science Education Resource Center. [Online]. Available: https://goo.gl/vsDCFB

[10] IRENA, Global Atlas for Renewable Energy: A World of Renewables. International Renewable Energy Agency, 2015. [Online]. Available: https://goo.gl/adpQop

[11] M. M. Riyahi Alam, A. Behfar, and R. Shahmoradi, "Potential application of solar power systems for residential buildings in high-density urban pattern: The case of the Eixample district, city of the Barcelona, in Spain," Recent Researches in Environmental and Geological Sciences, pp. 342-347, 2012. [Online]. Available: https://goo.gl/t6dSQZ

[12] IRENA, Renewable Energy in Cities. International Renewable Energy Agency, Abu Dhabi, 2016. [Online]. Available: https://goo.gl/EN2Ufq

[13] T. Razykov, C. Ferekides, D. Morel, E. Stefanakos, H. Ullal, and H. Upadhyaya, "Solar photovoltaic electricity: Current status and future prospects," Solar Energy, vol. 85, no. 8, pp. 1580-1608, 2011. [Online]. Available: https://doi.org/10.1016/j.solener.2010.12.002
[14] A. Barragán-Escandón, J. Terrados-Cepeda, E. Zalamea-León, and P. Arias-Reyes, "Electricity production using renewable resources in urban centres," Proceedings of the Institution of Civil Engineers - Energy, vol. 171, no. 1, pp. 12-25, 2018. [Online]. Available: https://doi.org/10.1680/jener.17.00003

[15] A. Curreli, G. Serra-Coch, A. Isalgue, I. Crespo, and H. Coch, "Solar energy as a form giver for future cities," Energies, vol. 9, no. 7, 2016. [Online]. Available: https://doi.org/10.3390/en9070544

[16] P. Chen, R. Salcedo, Q. Zhu, F. de Leon, D. Czarkowski, Z. Jiang, V. Spitsa, Z. Zabar, and R. E. Uosef, "Analysis of voltage profile problems due to the penetration of distributed generation in low-voltage secondary distribution networks," IEEE Transactions on Power Delivery, vol. 27, no. 4, pp. 2020-2028, Oct 2012. [Online]. Available: https://doi.org/10.1109/TPWRD.2012.2209684

[17] M. Wall, M. C. M. Probst, C. Roecker, M.-C. Dubois, M. Horvat, O. B. Jørgensen, and K. Kappel, "Achieving solar energy in architecture-IEA SHC Task 41," Energy Procedia, vol. 30, pp. 1250-1260, 2012. [Online]. Available: https://doi.org/10.1016/j.egypro.2012.11.138

[18] K. Farkas, F. Frontini, L. Maturi, A. Scognamiglio, M. C. Munari Probst, and C. Roecker, Designing photovoltaic systems for architectural integration. Criteria and guidelines for product and system developers, Solar Heating \& Cooling Programme- International Energy Agency, 2013. [Online]. Available: https://goo.gl/fj36VW

[19] B. P. Jelle, "Building integrated photovoltaics: A concise description of the current state of the art and possible research pathways," $E n$ ergies, vol. 9, no. 1, 2016. [Online]. Available: https://doi.org/10.3390/en9010021

[20] M. C. Munari Probst, C. Roecker, F. Frontini, A. Scognamiglio, K. Farkas, L. Maturi, and I. Zanetti, "Solar energy systems in architecture integration criteria and guidelines," Infoscience EPFL scientific publications, p. 214, 2013. [Online]. Available: https://goo.gl/4Rx7e5

[21] Eletrek. (2018) Tesla solar roof. [Online]. Available: https://goo.gl/Bnyduv

[22] A. Gharakhani Siraki and P. Pillay, "Comparison of PV system design software packages for urban applications," in Word Energy Congress Montreal, 2010. [Online]. Available: https://goo.gl/Vhuhyg

[23] G. Cáceres, S. Nasirov, H. Zhang, and G. Araya-Letelier, "Residential solar PV 
planning in Santiago, Chile: Incorporating the PM10 parameter," Sustainability, vol. 7, no. 1, pp. $722-440,2015$. [Online]. Available: https://doi.org/10.3390/su7010422

[24] S. C. S. Costa, A. S. A. C. Diniz, and L. L. Kazmerski, "Dust and soiling issues and impacts relating to solar energy systems: Literature review update for 2012-2015," Renewable and Sustainable Energy Reviews, vol. 63, pp. 33-61, 2016. [Online]. Available: https://doi.org/10.1016/j.rser.2016.04.059

[25] B. V. Chikate and Y. Sadawarte, "The factors affecting the performance of solar cell," International Journal of Computer Applications (0975-8887), 2015. [Online]. Available: https://goo.gl/d7txov

[26] M. E. Meral and F. Dinçer, "A review of the factors affecting operation and efficiency of photovoltaic based electricity generation systems," Renewable and Sustainable Energy Reviews, vol. 15, no. 5, pp. 2176-2184, 2011. [Online]. Available: https://doi.org/10.1016/j.rser.2011.01.010

[27] Y. Jia-Ying, D. Kun, T. Reindl, and A. G. Aberle, "Outdoor PV module performance under fluctuating irradiance conditions in tropical climates," Energy Procedia, vol. 33, pp. 238-247, 2013. [Online]. Available: https://doi.org/10.1016/j.egypro.2013.05.064

[28] A. Luque and S. Hegedus, Handbook of photovoltaic science and engineering. John Wiley \& Sons, 2011. [Online]. Available: https://goo.gl/7D9UYk

[29] C. Hachem, A. Athienitis, and P. Fazio, "Parametric investigation of geometric form effects on solar potential of housing units," Solar Energy, vol. 85, no. 9, pp. 1864-1877, 2011. [Online]. Available: https://doi.org/10.1016/j.solener.2011.04.027

[30] NREL. (2017) System Advisor Model (SAM). National Renewable Energy Laboratory. [Online]. Available: https://goo.gl/WTktPn

[31] J. Freeman, J. Whitmore, N. Blair, and A. P. Dobos, "Validation of multiple tools for flat plate photovoltaic modeling against measured data," in 2014 IEEE 40th Photovoltaic Specialist Conference (PVSC), June 2014, pp. 1932-1937. [Online]. Available: https://doi.org/10.1109/PVSC.2014.6925304

[32] I. F. Izquierdo Torres and M. G. Pacheco Portilla, Evaluación de la eficiencia de paneles solares como sistema de captación de energía para edificaciones del área urbana de Cuenca,
E. Universidad de Cuenca, Ed. Tesis de grado, 2017. [Online]. Available: https://goo.gl/wSEZ1X

[33] Climate-DATA. (2017) Clima Cuenca. climate-data.org. [Online]. Available: https: //goo.gl/SBnaa2

[34] J. Freeman, J. Whitmore, L. Kaffine, and A. P. Blair, Nate Dobos, "System Advisor Model: Flat plate photovoltaic performance modeling validation report," National Renewable Energy Laboratory (NREL), Tech. Rep., 2013. [Online]. Available: https://goo.gl/47cQ1r

[35] E. Rudié, A. Thornton, N. Rajendra, and S. Kerrigan, "System Advisor Model performance modeling validation report: Analysis of 100 sites," Locus Energy, National Renewable Energy Laboratory (NREL), Tech. Rep., 2014. [Online]. Available: https://goo.gl/zpfUZA

[36] D. F. Al Riza, S. Gilani, and M. Aris, "Measurement and simulation of standalone solar PV system for residential lighting in Malaysia," Journal of Hydrocarbons Mines and Environmental Research, vol. 2, no. 1, pp. 6-12, 2011. [Online]. Available: https://goo.gl/G8shmj

[37] K. Kanyarusoke, J. Gryzagoridis, and G. Oliver, "Validation of TRNSYS modelling for a fixed slope photovoltaic panel," Turkish Journal of Electrical Engineering \& Computer Sciences, vol. 24, no. 6 , pp. $4763-4772,2016$. [Online]. Available: https://www.doi.org/10.3906/elk-1502-38

[38] M. A. Meybodi, L. R. Santigosa, and A. C. Beath, "A study on the impact of time resolution in solar data on the performance modelling of CSP plants," Renewable Energy, vol. 109, pp. 551-563, 2017. [Online]. Available: https://doi.org/10.1016/j.renene.2017.03.024

[39] V. Håvard Breisnes, "Modelling of photovoltaic modules with battery energy storage in Simulink/MATLAB: With in-situ measurement comparisons," Master's thesis, Norwegian University of Science and Technology, 2014. [Online]. Available: https://goo.gl/13bxEy

[40] F. Chenlo Romero, "Cálculo de la temperatura de operación de células solares en un panel fotovoltaico plano," Centro de Investigaciones Energéticas, Medioambientales y Tecnológicas (CIEMAT). Madrid - España, Tech. Rep., 2002. [Online]. Available: https://goo.gl/SFrX4r

[41] M. Blumthaler, W. Ambach, and R. Ellinger, "Increase in solar UV radiation with altitude," Journal of Photochemistry and Photobiology B: Biology, vol. 39, no. 2, 
pp. 130-134, 1997. [Online]. Available: [43] E. Zalamea and R. García Alvarado, "Roof https://doi.org/10.1016/S1011-1344(96)00018-8

[42] E. F. Zalamea-León and R. H. García-Alvarado, "Integración de captación activa y pasiva en viviendas unifamiliares de emprendimientos inmobiliarios," Ambiente construido, vol. 18, no. 1, pp. 445-461, 2018. [Online]. Available: https: //dx.doi.org/10.1590/s1678-86212018000100231 characteristics for integrated solar collection in dwellings of Real-Estate developments in Concepción, Chile," Revista de la construcción, vol. 13, no. 3, pp. 36-44, 122014. [Online]. Available: https://dx.doi.org/10.4067/ S0718-915X2014000300005 